\title{
Development of the radio frequency quadrupole proton linac for ESS-Bilbao
}

\author{
Juan Luis Muñoz ${ }^{1 *}$, Ibon Bustinduy ${ }^{1}$, Igor Rueda ${ }^{1}$, and David de $\operatorname{Cos}^{1 \text { ** }}$ \\ ${ }^{1}$ ESS-Bilbao, Parque Tecnológico de Bizkaia, Laida Bidea, Edificio 207-B 48160 Derio, Bizkaia
}

\begin{abstract}
The Radio Frequency Quadrupole (RFQ) linear accelerator for ESS-Bilbao is described. This device will complete ESS-Bilbao injection chain after the ion source and the LEBT. The design was finished in 2015 and machining of the accelerator cavity started in 2016. The RFQ is a 4-vane structure, aimed to accelerate protons from $45 \mathrm{keV}$ to $3.0 \mathrm{MeV}$ and operating at $352.2 \mathrm{MHz}$ in pulsed mode with a duty cycle up to $10 \%$. Total length is about $3.1 \mathrm{~m}$ divided in 4 segments. Each segment is itself assembled from four components, named vanes, by using polymeric vacuum gaskets with no brazing among them. Notable aspects of the design are the constant mean aperture $R_{0}$, vane radius $\rho$ and thus $\rho / R_{0}$ ratio and also uniform inter vane voltage. Novel procedures for the design of the modulation and integrated beam dynamics and electromagnetic design have been developed for this task. In this paper, the complete design procedure and its results are presented, including beam dynamics, RF cavity design, field flatness and frequency tuning, cooling and thermo-mechanical design.
\end{abstract}

\section{Introduction}

ESS-Bilbao[1] is an Spanish public Consortium of the Central and Basque Governments. It is the institution selected to supply Spanish in-kind contribution to the European Spallation Source ERIC, ESS [2]. The contributions are focused in different areas: accelerator (the complete MEBT (Medium Energy Beam Transport) section and the RF systems of the warm section), target and neutron instruments (MIRACLES). In addition to these contributions, local projects are also under development. The ESS-Bilbao injector is a proton injector that consist on an ECR (Electron Cyclotron Resonance) ion source and a LEBT (Low Energy Beam Transport) system, already in operation[3]. This injector will be completed with the RFQ linac presented in this communication. The $3 \mathrm{MeV}$ can then be used as the linac of a compact acceleratordriven neutron source.

The ESS-Bilbao RFQ design was carried out by a local team. The ISIS-FETS[4] and Linac4[5] (also ESS[6], based on IPHI[7]) RFQs were taken as references and initial state-of-the-art models. However, the frozen design incorporates mixed selection of characteristics. The rounded lobe shape (the so-called Montgolfier shape), external geometric characteristics and, more importantly, the assembly procedure without brazing were adapted from ISIS-FETS. The tuning approach (based on movable tuners) and the cooling strategy of the FETS RFQ were replaced by more standard cooling based on longitudinal drilled channels in the vane, and fine tuning by cooling water temperature. But due to the external characteristics (two minor and two

\footnotetext{
*e-mail: jlmunoz@essbilbao.org

** Now at Departamento de Física Aplicada II, Facultad de Farmacia, Universidad del País Vasco, Paseo de la Universidad 7, 01006 VitoriaGasteiz, Spain
}

major vanes with vacuum pump grids) there are no longitudinal cooling channels in the body of the cavity, so the approach to tuning is different than in other RFQs. The complete design process has been collected in a Technical Design Report (TDR)[8]. This paper discusses the different approaches and the design route chosen and the corresponding tools that have been used. The conclusions can be useful to other groups attempting to design an RFQ linac for use in a CANS facility.

The non-conventional approach to the design of the modulation and the resonant cavity needed the design of home-made computer tools or to adapt existing ones. The software used for each design step will be described in the corresponding section. The RFQ is currently under fabrication, and first tests are expected to start during 2019.

The RFQ is divided in four segments of around $800 \mathrm{~mm}$ in length. Each segment is itself an assembly of four components, two major vanes and two minor vanes, as depicted in Fig. 1. There are no coupling structures between segments. The vanes are assembled by means of polymeric vacuum gaskets (3D O-rings), using no brazing or welding. Finger strips are also added in all copper contact surfaces to improve RF contact. This strategy was adapted from the ISIS-FETS RFQ[4], and has been also proposed for other projects[9]. The vacuum ports location (in the upper and lower major vanes) is also taken from FETS RFQ. Cooling scheme is different from this RFQ though, as drilled channels instead of cooling-pockets, are used for the vacuum grid and along the vane tips.

Cavity tuning and field stabilization will be provided by static plunger tuners, while cooling water temperature will be used to fine tune the RFQ operation. Movable plunger tuners can, nonetheless, be used if needed. Cooling channels are drilled only along the vane tips (and not in the structure body). This was decided by mechanical and 


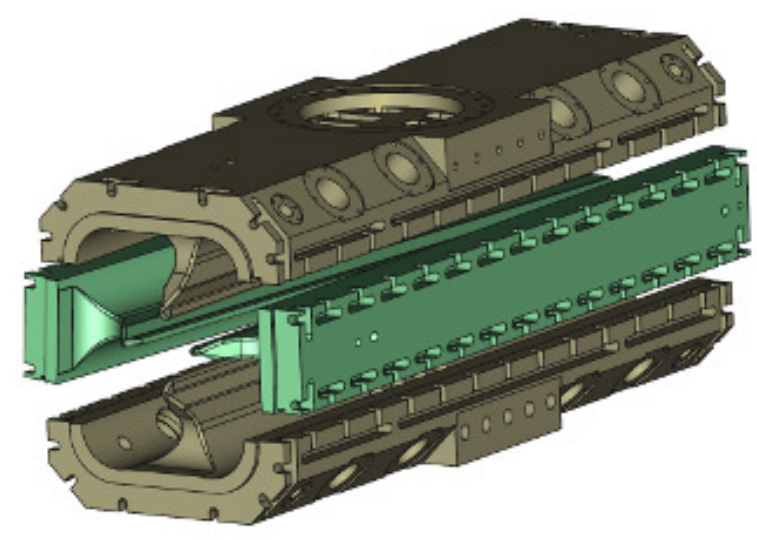

Figure 1. Exploded view of first segment of the RFQ, showing the so-called major and minor vanes.

Table 1. ESS-Bilbao RFQ Main Characteristics.

\begin{tabular}{lc}
\hline & ESS-Bilbao RFQ \\
\hline Type & 4 vane \\
Particle & Protons \\
RF frequency & $352.2 \mathrm{MHz}$ \\
Innervate Voltage & $85 \mathrm{kV}$ (uniform) \\
Energy & $45 \mathrm{keV} \longrightarrow 3.0 \mathrm{MeV}$ \\
Design current & $60 \mathrm{~mA}$ \\
Input emittance (rms norm) & $0.25 \pi \mathrm{mm} \mathrm{mrad}$ \\
Duty cycle & Up to $10 \%$ \\
Kilpatrick factor & 1.85 \\
Number of cells & 273 \\
$R_{0}$ & $3.44 \mathrm{~mm}$ \\
$\rho / R_{0}$ & 0.85 \\
Input/Output matcher & $16.674 \mathrm{~mm} / 14 \mathrm{~mm}$ \\
Total length & $3.12 \mathrm{~m}(3.66 \lambda)$ \\
Number of segments & 4 (about $800 \mathrm{~mm}$ each) \\
Method of assembly & Polymeric gaskets + RF seals \\
Plunger tuner ports & 16 per segment \\
\hline
\end{tabular}

fabrication criteria. This means that the frequency control by the cooling water temperature will operate only on vane-tip channels, contrary to other RFQs where two water circuits (vane tip and body) are available for tuning ([5-7, 10, 11]). Extensive thermo-mechanical and electromagnetic simulation studies have been done to proof that the operational control can be performed this way. Tests with the first segment will verify this approach.

The RFQ is designed to accelerate protons from $45 \mathrm{keV}$ to $3.0 \mathrm{MeV}$. It is a pulsed machine, operating at $352.2 \mathrm{MHz}$ with an expected duty cycle in operation of $5 \%$ (designed up to $10 \%$ ). Notable aspects of the design are the constant mean aperture $R_{0}$, vane radius $\rho$ and thus $\rho / R_{0}$ ratio. Inter-vane voltage is also uniform, with a value of $85 \mathrm{kV}$. Main characteristics of the RFQ can be found in Table 1.

In each segment there are 16 tuner ports $(\varnothing 37 \mathrm{~mm})$ that can be used for static plunger tuners. The power coupler flange also fits in these ports, so that they can be inserted in any position. Additionally, $8 \varnothing 16 \mathrm{~mm}$ ports are built in order to be used for pick-ups or other sensors needed at any moment. Vacuum grid ports are designed for standard $\varnothing 210 \mathrm{~mm}$ flanges. All ports are machined in the major vanes.

\section{MODULATION AND BEAM DYNAMICS}

\subsection{Modulation design}

The ESS-Bilbao RFQ modulation is the result of an optimization process. The modulation is designed for an inter-vane voltage of $85 \mathrm{kV}$, uniform throughout the entire length. Vane radius $(\rho)$ is also constant, so to obtain a uniform local frequency and field flatness the mean aperture $R_{0}$ should also be constant. Modulation follows the shape of a 2-term expansion of the inter-vane voltage[12] and has been designed using a modified version of RFQSIM code[13].

The aim of the modulation design was to obtain an RFQ shorter than $4 \lambda=3.4 \mathrm{~m}$ to simplify tuning operations. Also, copper blocks $800 \mathrm{~mm}$ long were already available, so an additional target was to fit the total length of the RFQ in four segments, instead of five, reducing inter segments joints and the risks associated. A final length limit of $3.1 \mathrm{~m}$ was selected.

Another figure of merit target for optimization was the Kilpatrick factor (ratio of designed maximum electric field value with respect to the theoretical maximum for copper at the operating frequency), that was chosen to be below 1.85 .

\subsubsection{Modulation optimization process}

The optimization procedure starts by obtaining a modulation geometry, shorter than the maximum length allowed, that fulfils the beam dynamics criteria (transmision and beam losses). The main tool for this task is a modified version of RFQSIM code that uses a 2-term voltage expansion of the vanes geometry. Electromagnetic calculations for surface electric field and RF electromagnetic field for particle tracking were then performed. These computations used the actual geometry of the vane tips in FEM codes. The initial parameters were then modified according to the FEM results, and a new optimization run starts. This procedure continues until an optimal solution is obtained.

The input parameters that define a particular modulation are (using the standard parameter naming convention [14]): synchronous phases and particle energy at the end of each section (Shaper $\left(\phi_{\mathrm{sh}}, W_{\mathrm{sh}}\right)$, Gentle Buncher $\left(\phi_{\mathrm{gb}}, W_{\mathrm{gb}}\right)$ and Accelerator $\left.\left(\phi_{\mathrm{f}}, W_{\mathrm{f}}\right)\right)$, clear aperture $\left(a_{\mathrm{gb}}\right)$ and modulation $\left.\left(m_{\mathrm{gb}}\right)\right)$ parameters, and input and output matcher sections radius.

In order to obtain a short modulation the range of parameters during optimization was oriented towards this target, with preference over other goals such as maximizing the transmission or maintaining a conservative Kilpatrick factor. In this sense, we concentrated the parameter space search on areas with lower clear aperture $a_{\mathrm{gb}}$ 


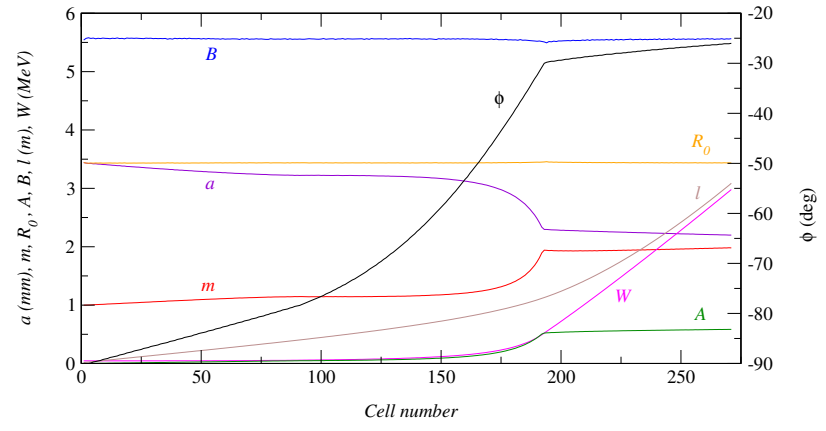

Figure 2. Evolution of several modulation parameters as a function of the cell number.

and energy $W_{\mathrm{gb}}$, and less negative synchronous phase $\phi_{\mathrm{gb}}$ (all referred to the end of the Gentle Buncher). Particle tracking results obtained with RFQSIM presented transmissions below $90 \%$ for modulations found in this region of the parameter space. However, later simulations with other codes presented much better results. This strategy also produced higher surface fields, with a peak at the end of the Shaper typically above 1.8 times the Kilpatrick limit.

Also, a progressive reduction of the aperture in the Acceleration section was implemented in order to shorten the modulation. RFQSIM originally built the 2-term based Acceleration section using a constant aperture and modulation factor, following the rules proposed by Kapchinskiy-Tepliakov[12]. This produces slowly decaying fields along $z$, due to the increased cell length. We modified the part of RFQSIM that creates the 2-term modulation to incorporate custom aperture reduction strategies in the Acceleration section, as well as the ability to set a goal total length for the modulation.

The evolution of the main parameters as a function of cell number is shown in Fig. 2, while the final modulation shape and the corresponding accelerating field are shown in Fig. 3.

Concerning the high energy end of the modulation, it has been designed with the aim of optimizing the transmission through the designed MEBT and DTL. After the last regular cell of the accelerator section, a transition cell is included so both $X$ and $Y$ vanes end with the same aperture. A circular output matcher ends the modulation. The transition cell has a length of $17 \mathrm{~mm}$ and the output matcher has a radius of $14 \mathrm{~mm}$. The last cells are shown in Fig. 4.

\subsubsection{Surface field calculations}

Although RFQ design software packages provide a calculation of Kilpatrick factor (surface electric field) we preferred to perform these calculations in an external finite element software, in order to gain control and accuracy over the process. The modulation description provided by RFQSIM consist on the modulation amplitude at the beginning and end of each cell (of length $\beta \lambda / 2$ ). The actual shape of the modulation spine is built cell by cell by performing the 2-term interpolation in a Matlab script. The output of this script is a pair of curves $V_{x}(z)$ and $V_{y}(z)$ for horizontal and vertical vanes, respectively. The cell 3D geometry vane region is then built as a parametric surface for each vane in COMSOL Multiphysics FEM software. For example, the geometry of the profile of an horizontal vane cell between $z$ coordinates $z_{0}$ and $z_{1}$ is defined as a parametric surface dependent on parameters $u \in[0,1]$ and $v \in[-\pi / 2, \pi / 2]$ as defined in Eq. 1. Vertical vanes are defined in a similar way.

$$
\left\{\begin{array}{l}
x=V_{x}(z(u))+\rho(1-\sin (v)) \\
y=\rho \sin (v) \\
z=z_{0}+u\left(z_{1}-z_{0}\right)
\end{array}\right.
$$

The surface field is computed running an electrostatics simulation. To avoid border effects the model is built for three consecutive cells, but only the results for the central one are considered. The process is fully automated using Matlab/COMSOL scripts. The result for a particular modulation is the curve $E_{s, \max }(z)$, that is the maximum value of the surface field for the cell starting at coordinate $z$. The overall maximum value of this curve is taken as the figure of merit characterizing the particular modulation. Using this approach we managed to scan a huge and fine set of parameter ranges in an automatic, brute-force approach. An example of such electrostatics model is shown in Fig. 5 .

The results obtained for the final modulation are shown in Fig. 6. The peak that can be seen at around $z=0.5 \mathrm{~m}$ is caused by the transition between Shaper and Gentle Buncher sections, while the gradual increase from $z=$ $1.3 \mathrm{~m}$ is a consequence of designing for higher accelerating fields in order to reduce modulation length.

Surface field calculations have also been used to determine the shape and position of gaps between the segments. The modulation is described as a continuous function from the beginning of the input matcher to the end of the output matcher. But the segmentation of the RFQ structure forces cuts in the modulation at certain $z$ coordinates corresponding to the end of each segment. In order to reduce the perturbation that these cuts cause on the electric field, extensive simulation studies were made[8]. In these studies the position of the end-beginning of segments were considered between the cell-end position and the Lloyd position [15]. In the first case the longitudinal accelerating field is zero for all particles in the gap, but not the transverse components. In the second case, the field vanishes when the bunch center crosses the gap. Also, the rounding of the vane tips in the cut was studied, finally selecting an elliptical shape instead of a circular one. These studies included surface field calculation and also beam dynamics simulations. The overall best results, considering figures of merit and segment mechanical length conclusion was to use gaps $200 \mu \mathrm{m}$ long placed at the end-cell position; the end of the vanes are rounded in an elliptical shape with long semi-axes of $2 \mathrm{~mm}$ length and short semi-axes having $0.75 \mathrm{~mm}$ (see Fig. 7). 

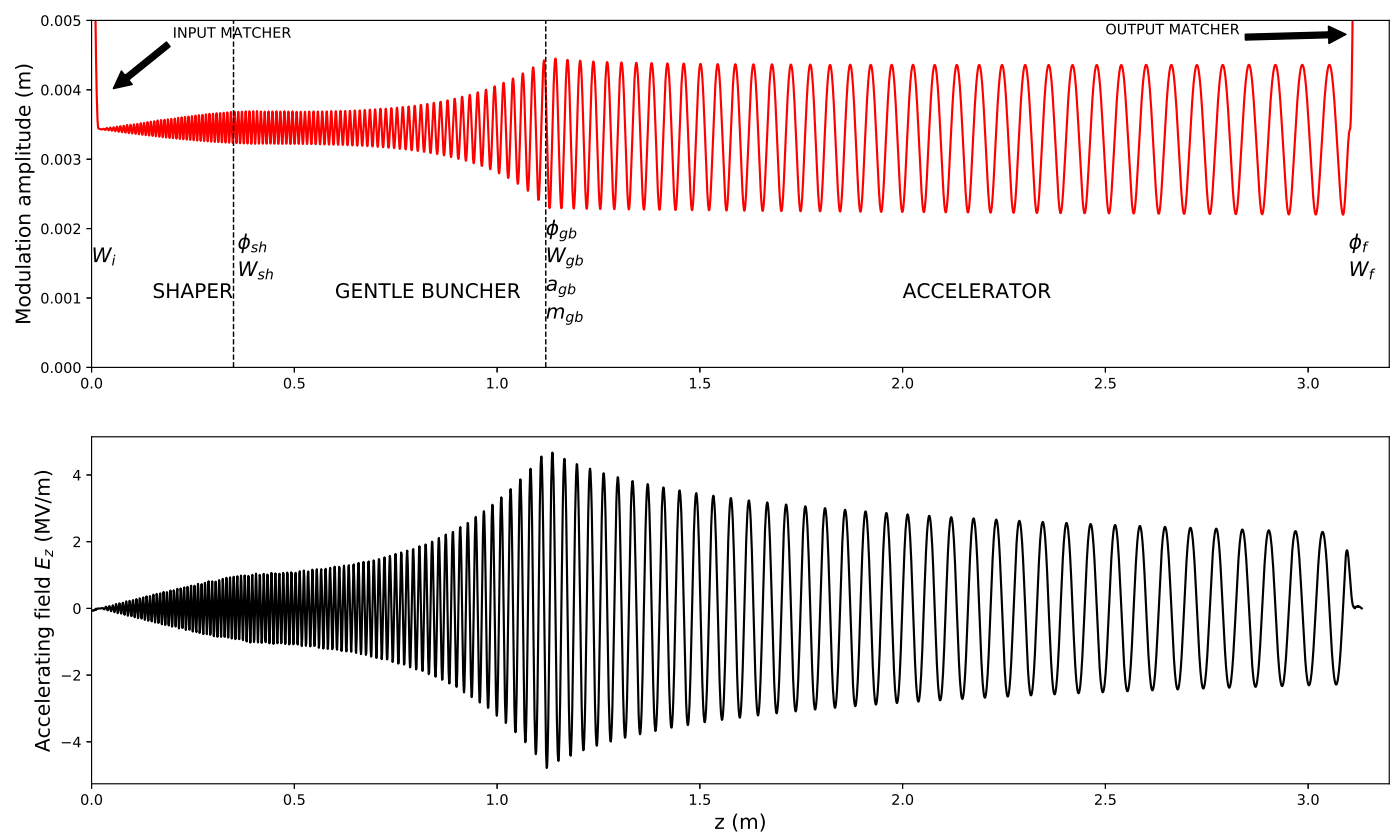

Figure 3. Modulation amplitude and accelerating field for ESS-Bilbao RFQ.

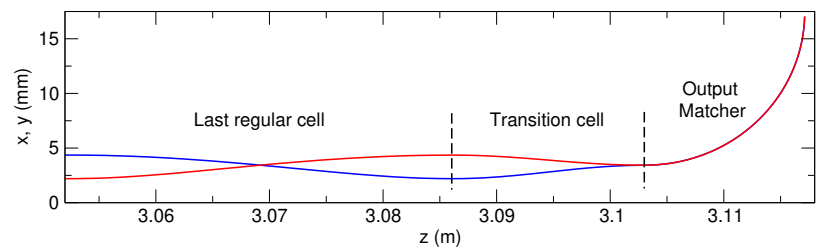

Figure 4. Characteristics of the last cells of the RFQ.

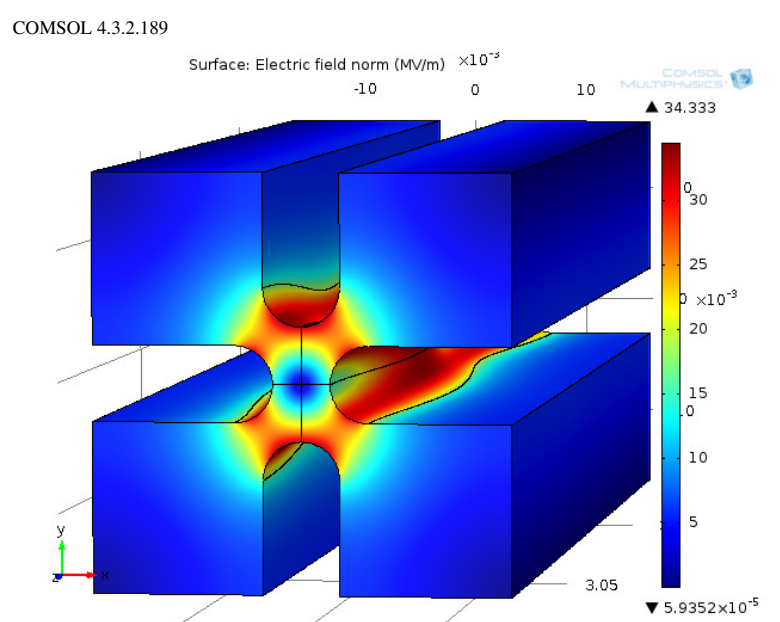

Figure 5. Surface electric field in an electrostatics model of three cells computed to extract the maximum surface field per cell. Only the vane region is considered for the calculation.

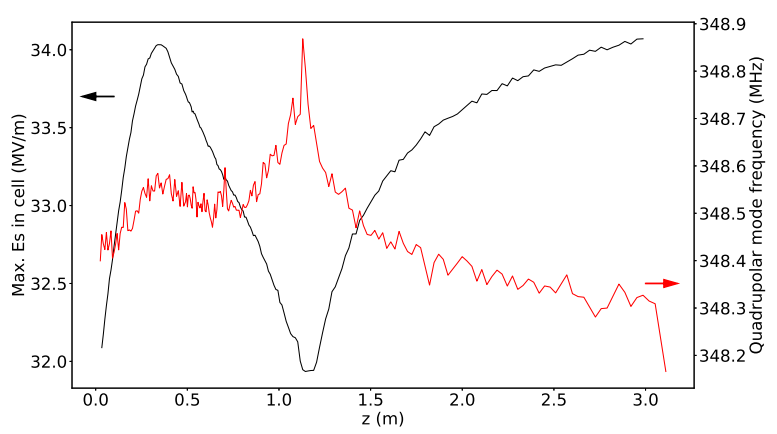

Figure 6. Surface maximum electric field per cell (left) and local frequency (right) as a function of cell starting coordinate $z$. Frequency is calculated in a slice of RFQ with one cell length and no tuners.

\subsection{Beam dynamics results}

From the modulation description obtained by using the optimization procedure, the full-vane 3D geometry of the RFQ was built in COMSOL Multiphysics software. Electrostatic simulations were run and the electric field was then exported in an external file. Particle tracking analysis using GPT[16] code were then run with the precise field map as input. Several optimization runs were made, exploring different regions of the parameter space until the final modulation was selected. Results were then crosschecked using different codes (Toutatis[17], PARMTEQ 


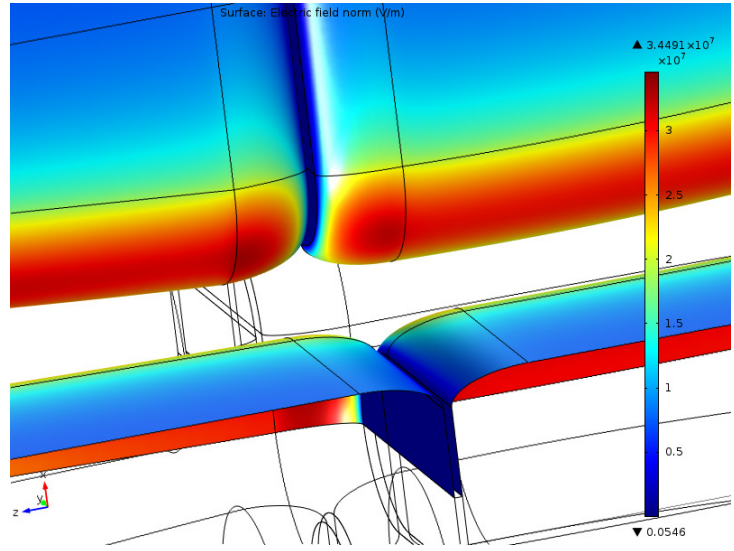

Figure 7. Electric field in the surface of vane tips around a gap between segments. The shape of the end of the vanes is elliptical.

Table 2. Simulation results at the output of the RFQ for the four methods used. The figures of merit are: full transmission (\%), accelerated transmission (\%), transverse normalized rms emittance ( $\pi \mathrm{mm}$ mrad) calculated as the average between the horizontal and the vertical values, longitudinal rms emittance $(\pi \operatorname{deg} \mathrm{MeV})$ and maximum surface electric field $(\mathrm{MV} / \mathrm{m})$.

\begin{tabular}{lcrccl}
\hline Code & $T_{\text {all }}$ & $T_{\text {good }}$ & $\epsilon_{t}$ & $\epsilon_{z}$ & $E_{s}$ \\
\hline RFQSIM & 90.7 & 86.8 & 0.243 & 0.120 & 30.4 \\
TOUTATIS & 94.6 & 93.6 & 0.266 & 0.131 & 33.4 \\
GPT + COMSOL & 95.2 & 94.1 & 0.264 & - & 34.1 \\
RFQGen & 96.2 & 94.1 & 0.241 & 0.125 & 33.7 \\
\hline
\end{tabular}

/ RFQGen). Additional characteristics of the modulation can also be found in Table 1 .

Beam dynamics simulations have been performed using different codes for comparison. All the simulations were performed using the same input beam characteristics: $45 \mathrm{kV}$ input beam energy, $60 \mathrm{~mA}$ current intensity and $0.25 \pi \mathrm{mm}$ mrad transverse emittance. The C-S (CourantSnyder[14]) of the input beam varied slightly from code to code, depending on the optimal match calculated in each case, with values of alpha typically a little above 1 , and beta about $0.03 \mathrm{~m} / \mathrm{rad}$.

The codes used for comparison of results were: GPT + COMSOL, RFQSIM, TOUTATIS and RFQGen (an improved fork of PARMTEQ). The implementation of our design in RFQGen is not completely accurate, since this code did not include an option to import a cell-by-cell description of the modulation at the time. So in consequence, the current RFQGen results must be considered as approximate.

Table 2 presents the main results of the particle tracking simulations. The accelerated $(\sim 3 \mathrm{MeV})$ beam transmissions of TOUTATIS, GPT + COMSOL and RFQGen are almost identical $(\sim 94 \%)$. The emittance results are very positive, with virtually null increase of the transverse emittance, and a value of $0.12-0.13 \mathrm{deg} \mathrm{MeV}$ for the longitudinal emittance.The surface fields, while not identical (they are calculated by different approaches), lie within $1.81-1.85$ times the Kilpatrick limit $\left(18.47 \mathrm{MV} \mathrm{m}^{-1}\right.$ for $352.2 \mathrm{MHz})$.

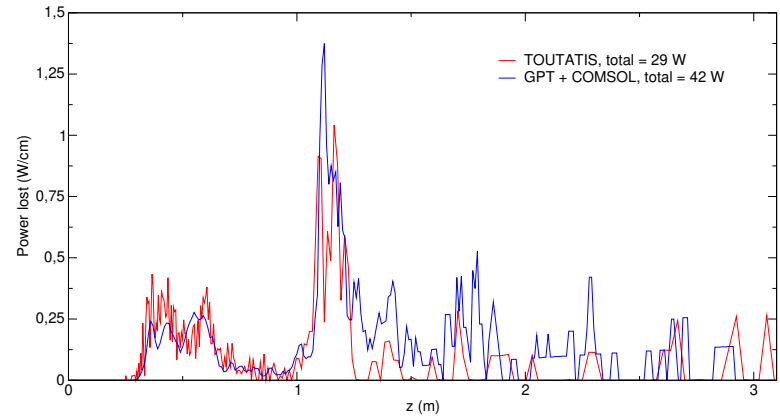

Figure 8. Beam power loss per centimeter of RFQ length, simulated with two different methods.

Results obtained with RFQSIM, however, have a poorer transmission than the other three codes, which is mainly due to a predicted transverse loss of almost $10 \%$ of the beam. This is due to the method of field calculation in each code [18]. The other three codes represent the actual transmission of the RFQ more accurately, given that TOUTATIS and RFQGen (PARMTEQ) are the most widely used codes in the field of RFQ beam dynamic and that the field maps provided by COMSOL comes from a finite element simulation with accurate representation of 3D vane geometry, and the tracking is performed with GPT, a code extensively used beam dynamics simulations of other accelerator elements.

Fig. 8 shows the transverse losses (due to particles impacting with the vanes) along the RFQ, by depicting the beam power lost per centimeter of length. The curves are very similar in the Shaper and Gentle Buncher regions (first $1.2 \mathrm{~m}$ ), with slightly higher power loss predicted by GPT + COMSOL in the Acceleration section. The total power lost (integral of the curves) remains within reasonable values in both cases, since most of the lost particles impact the vanes with energies in the hundreds of $\mathrm{keV}$.

In summary, the results presented above prove the validity of the proposed vane modulation in terms of beam dynamics, as tested with different particle tracking and field map calculating methods. Although some of the new design constraints (especially the $\sim 20 \%$ length reduction) were initially expected to reflect negatively on the beam transport performance, this is only apparent in the RFQSIM results, with the other three codes presenting transmissions of $\sim 94 \%$.

\subsection{Local frequency profile}

The vane modulation changes the local capacitance per unit length of the ideal quadrupole resonator. As a consequence there is a perturbation on the voltage profile $V(z)$, that no longer is constant. This perturbation can be modeled by considering a local resonant frequency computed in a cell-by-cell approach. Considering a modulation cell that is located between $z_{i}$ and $z_{i+1}$, the local frequency $f_{q}\left(z_{i}\right)$ is the frequency of the resonator built from a slice of RFQ volume between $z_{i}$ and $z_{i+1}$, considering perpendicular magnetic field boundary conditions at both ends and 
perfect electric boundary condition at the rest of the surfaces. This frequency profile should be as flat as possible because voltage profile in the real cavity will be dependent on this perturbation[14]. For the first (2013) design of ESS-Bilbao RFQ a sinusoidal (instead of 2-term) vane profile was used. This resulted in a very perturbed frequency profile[8]. For the final design a 2-term modulation shape (meaning that the geometry mimics the profile of an expansion of voltage truncated to two terms) was used. This resulted in the rather flat profile shown in Fig. 6. Deviation of local frequency around the 2D ideal design frequency is of the order of $0.1 \mathrm{MHz}$, while with a sinusoidal shape it was of several $\mathrm{MHz}$ (see the TDR[8] for details).

\section{CAVITY DESIGN}

\subsection{Cavity cross-section design}

The cross section of the cavity is based on the circular lobe approach used by ISIS-FETS[4], but using straight (vertical or horizontal) segments in the vanes, to aid the alignment verification operations later on. The machining of the rounded shape of the lobes was tested by fabricating different models in copper and aluminum[19]. The cross section is uniform for all the length of the RFQ. Although a variable lobe diameter along length is common in the current state-of-the-art, the uniform approach was selected as a conservative decision concerning mechanical engineering. For future developments, a variable approach would be probably preferred.

The optimization of the geometry was made by parametric simulations. The optimum values were selected by minimization of the power losses (or maximization of the quality factor, $Q_{0}$ ). The range of parameters available during the optimization procedure was restricted by mechanical constraints, like the total width of copper blocks, cooling channels diameter and corresponding wall thickness. In the optimization process, one of the parameters was always free, so it could be adjusted later to obtain the right frequency. The design frequency for the $2 \mathrm{D}$ models was chosen to be $348.6 \mathrm{MHz}$ (several $\mathrm{MHz}$ below operating frequency) to avoid problems due to machining. The frequency will be raised to the operational one by means of plunger tuners. The average modulation aperture $(3.438 \mathrm{~mm})$ was used as vane tip aperture in the $2 \mathrm{D}$ models. A sketch of the cross-section is shown in Fig. 9, while in Fig. 10 electric and magnetic field maps for whole cross section in the quadrupolar mode are shown.

\subsection{D cavity design}

The 3D body of the cavity is constructed by extruding the cross-section, adding the vane modulation solids, the input and output regions and other details like tuner and vacuum ports. Again, the shape has been optimized by finite element simulations aiming at reducing power deposition. An overall longitudinal cross section is shown in Fig. 11. The whole structure is an assembly of the four segments.

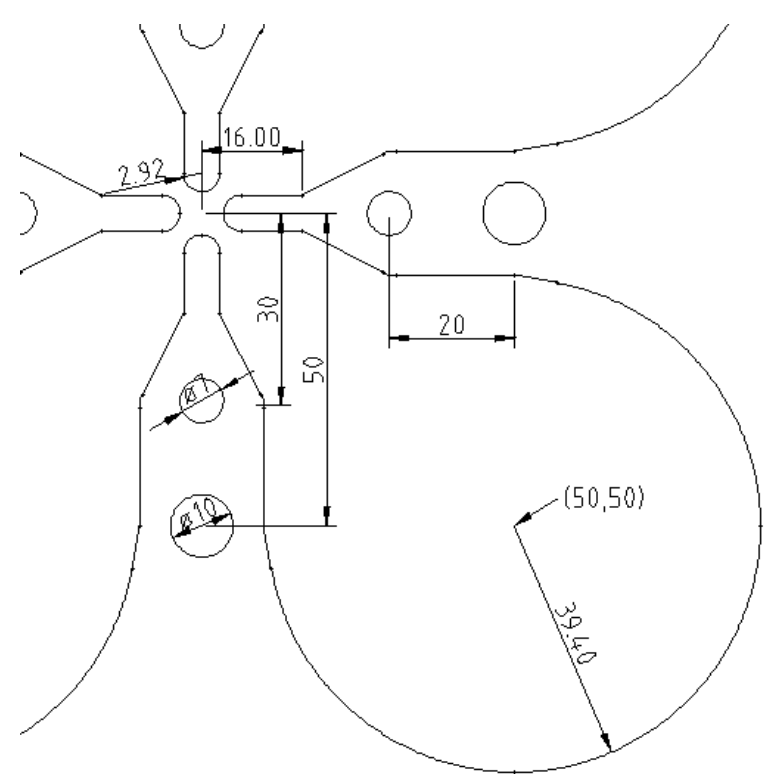

Figure 9. Sketch of a portion of the RFQ cross section, showing some dimensions (all in $\mathrm{mm}$ ). The vane tips geometry comes from the average values of the modulation and the cooling channels position and diameter, that restrict vane thickness due to impose a minimum wall thickness, are fixed values. All the other parameters were subject of optimization.

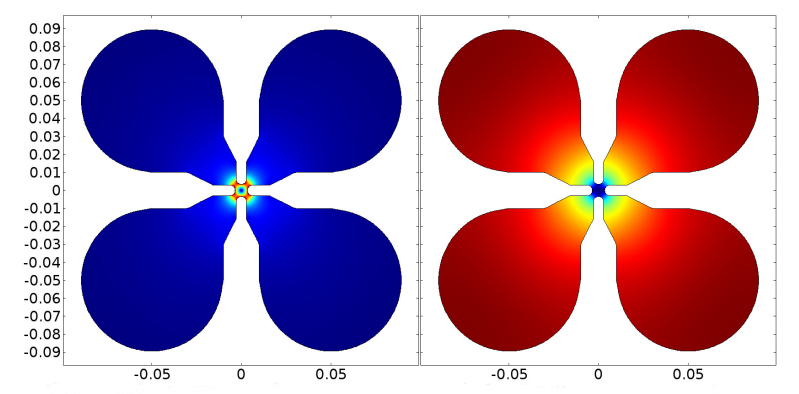

Figure 10. Cross section of the RFQ, showing normalized electric (left) and magnetic (right) field distribution for the quadrupolar mode.

Each segment has its own set of channels for cooling of the vanes and for cooling of the vacuum grid region.

The input and output undercut sections were designed so they have the same frequency as the bulk of the RFQ, contributing in this way to maintain the field flatness. The optimization looked for the optimum set of parameters that reduced power losses and kept electric voltage flatness. An schematics of the input section with the optimization parameters is shown in Fig. 12. Parameter values can be found in Table 3.

The field flatness was verified by $3 \mathrm{D}$ simulations of the whole length of the RFQ (not only the input or output undercut regions), and by mathematical transmission line models of the structure. One of the parameters is the distance between the vane tips and input (or output) lid (see Fig. 12). This distance can be easily modified after fabrication and testing of the RFQ, as it only involves changes in the lid, and not in the main body of the cavity. 


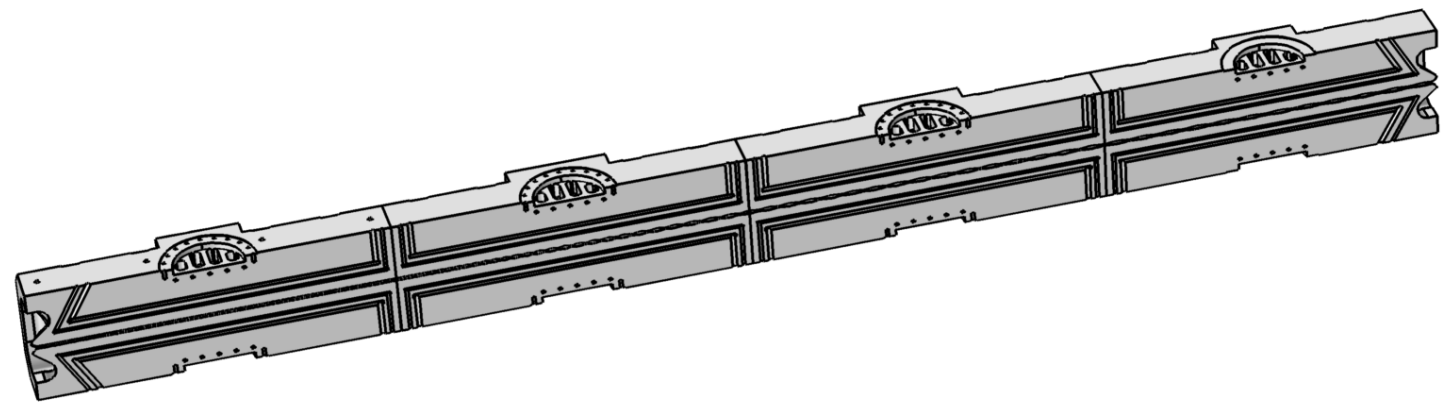

Figure 11. Longitudinal cross section of the RFQ structure. The four segments can be seen, showing the cooling channels and the vacuum ports.

Table 3. Parameters for input and output sections of the RFQ. Refer to Fig. 12 for description of parameters.

\begin{tabular}{lcc}
\hline Parameter & Input section & Output section \\
\hline$R_{\text {matcher }}[\mathrm{mm}]$ & 16.674 & 14.0 \\
$z_{\text {cover }}[\mathrm{mm}]$ & 7.5 & 7.5 \\
$x y_{\text {lobe }}[\mathrm{mm}]$ & 88 & 88 \\
$r_{\text {matcher }}[\mathrm{mm}]$ & 25 & 25 \\
$z_{\text {matcher }}[\mathrm{mm}]$ & 22.60 & 21.0 \\
\hline
\end{tabular}

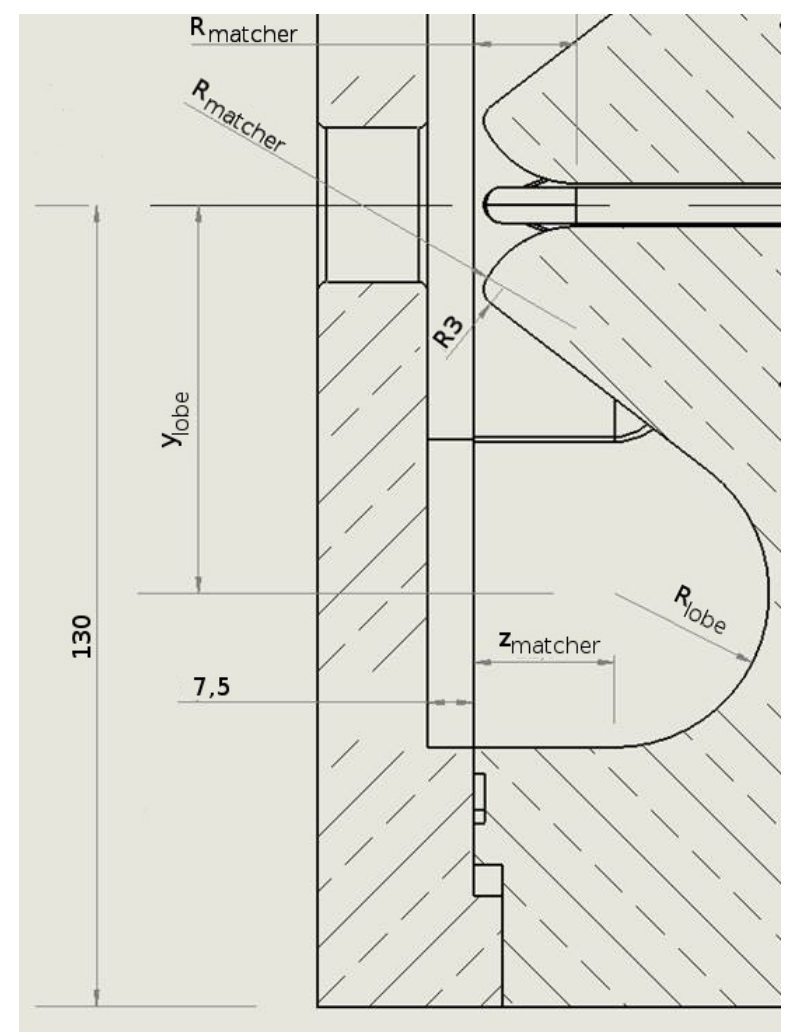

Figure 12. Schematics of the input undercut section of the RFQ, showing the parameters chosen for optimization.
The vacuum port grid also changes slightly the local frequency of the cavity. In order to compensate this detuning, the solid part of the grid penetrates the cavity as ridges, that have been also designed to provide the right frequency. These ridges are cooled by the vacuum port cooling channels. The ridges have been rounded to facilitate the machining process.

\section{3D ELECTROMAGNETIC SIMULATIONS AND TUNING}

Electromagnetic FEM simulations of the whole length of the RFQ including the details of the modulation and undercuts have been performed to verify the design. These simulations require a lot of resources in preparation of the models and computation time. For this purpose, models with a simplified geometries were used whenever possible. Simulations have been solved efficiently using COMSOL Multiphysics eigenvalue solver. A more detailed description can be found elsewhere [20].

The vane tip region must be defined very finely in order to catch all the details of the modulation. The geometric construction of the vanes themselves is challenging, and we couldn't do it in a reliable way using the CAD packages that we had available, so a home made software that makes use of OpenCascade 3D technology[21] was coded and used for this. The tool allows to import the file describing the modulation for the whole length with a resolution of 50 points per cell. The vane modulation CAD files produced this way were also used for the final solids supplied to the manufacturer.

The electrostatics ( $E S$ ) simulations with constant voltage boundary condition were used during the design of the RFQ modulation. ES simulations were also used to scale the electromagnetic fields obtained in an eigenvalue simulation (that have no power input reference). This procedure is detailed in [20]. An example of the results of the $3 \mathrm{D}$ simulations is shown in figure 13 , where power losses 
Table 4. Frequency spectrum of the RFQ (in MHz), computed from FEM simulations of the whole structure.

\begin{tabular}{cccc}
\hline Mode index & Q & D1 & D2 \\
\hline 0 & 351,9651 & 339,9509 & 339,9814 \\
1 & 355,2243 & 346,2343 & 346,309 \\
2 & 364,8562 & 356,3018 & 356,423 \\
\hline
\end{tabular}

computed from the fields in the surface, and adequately scaled to an innervate voltage of $85 \mathrm{kV}$, are depicted.

A mathematical model based on a transmission line description of the RFQ cavity[14] has also been developed. The model has been adjusted using the FEM calculation results and implemented in a computer tool to assist in the tuning operations. A comparison between the innervate voltage computed with the transmission line model and extracted from the FEM models is shown in Fig. 14.

\subsection{Mode spectrum}

From the 3D computer simulations of the whole length the frequency spectrum of the RFQ cavity can be obtained. Previously, this was estimated by extrapolation from only first segment simulations. Computed modes frequencies are grouped in table 4. Modes near the first quadrupolar one are well separated in frequency. This will contribute to an easy tuning and stabilization of the RFQ.

\subsection{Static tuning}

Static tuning of the RFQ will be provided by a set of plunger tuners. For each $800 \mathrm{~mm}$ segment there are 4 sets of 4 tuner ports, so a maximum of 64 tuners could be installed in the whole length (two ports will be used by the power couplers). The static tuners will increase the cavity frequency from the $348.6 \mathrm{MHz}$ of the design to a value close to the operational frequency of $352.21 \mathrm{MHz}$. The voltage profile (field flatness) should be kept as uniform as possible, and this will probably require that the tuners penetrate the cavity in a non-uniform way. The combination of FEM and mathematical models will be used for this once the actual voltage profile is measured in the final cavity. An automatic procedure will calculate the tuners penetration that correct the measured profile and result in a flat one. This has been implemented in a software tool and tested with numerical examples, as shown in Fig. 15.

\section{COOLING DESIGN AND DYNAMIC TUNING}

The RFQ is water cooled. The cooling removes the excess heat and also is used to fine tune the RFQ cavity during operation by controlling the thermal expansion driven frequency changes. For each segment, there are cooling channels near the vanes and also in the vacuum grid area.

\subsection{Heat load}

The heat load coming from the RF standing wave excited to the RFQ cavity walls is computed from the electromagnetic fields at the surface $[14,20]$, using a copper surface resistance of $R_{s}=0.0052 \Omega$. The eigenfrequency problem is solved and the fields scaled as previously described. A power loss of about $17 \mathrm{~kW}$ is estimated at $5 \%$ duty cycle.

\subsection{Cooling channels and thermo-mechanical results}

There are two types of cooling channels in the structure. Longitudinal channels run along the vane tips (with inlets and outlets in the external surface), while transversal channels cool the vacuum pump grid region. These channels can be seen in Fig. 16.

Thermo-mechanical simulations have been done using a simplified geometry, in order to increase the speed particularly for transient simulations. Simulations are done also using COMSOL Multiphysics, taking the power losses density maps as input heat flux in the internal surfaces. Coupled heat transfer, CFD and thermo-mechanical models of the cavity are then solved. The deformation of the vacuum region of the cavity allows to compute again the resonant frequency. In this way, the detuning caused by the thermo-mechanical deformations can be studied in any condition: steady state or transient simulations, with different RF duty cycles as power inputs and different water input temperatures and flows.

An example of the temperature distribution in the solid and the mechanical deformation can be seen in Fig. 16. For a duty cycle of the $5 \%$ and the temperature of the input water fixed at $25^{\circ} \mathrm{C}$, the temperature of the copper is below $31^{\circ} \mathrm{C}$. In Fig. 17 the dependence of cavity frequency (first segment) with cooling water temperature is shown for steady state simulations. The detuning is very small (about $19 \mathrm{kHz} /{ }^{\circ} \mathrm{C}$. This is due to the thickness of the walls. A similar detuning of $9.3 \mathrm{kHz} /$ duty is obtained by changing the duty cycle percentage. These results point out the stability of the operation when an adequate water flow is used.

Transient conditions have also studied this way, in order to study the fine control of frequency during operation of the cavity. As an example of these calculations, in Fig. 18 the dynamic change of frequency during a power on step (and the opposite power off step) is shown. These results are used to tune a control model of the cavity frequency, where a mixture of cold and warm water can stabilize the frequency of the RFQ easily. These results are beyond the scope of this paper and will be duly presented elsewhere. Additionally, movable plunger tuners can be used at certain lengths if needed. One plunger tuner per quadrant will provide a tuning range of $20 \mathrm{kHz} / \mathrm{mm}$, enough for tuning the cavity in a LLRF loop. This solution is a back-up plan.

\section{FABRICATION}

The fabrication of the first segment of ESS-Bilbao RFQ started in 2016. As mentioned before, the RFQ is a 4vane structure. It has a total length of about 3.1 meters, divided in 4 segments of about $800 \mathrm{~mm}$ length each. The segment length is determined by the machining equipment 


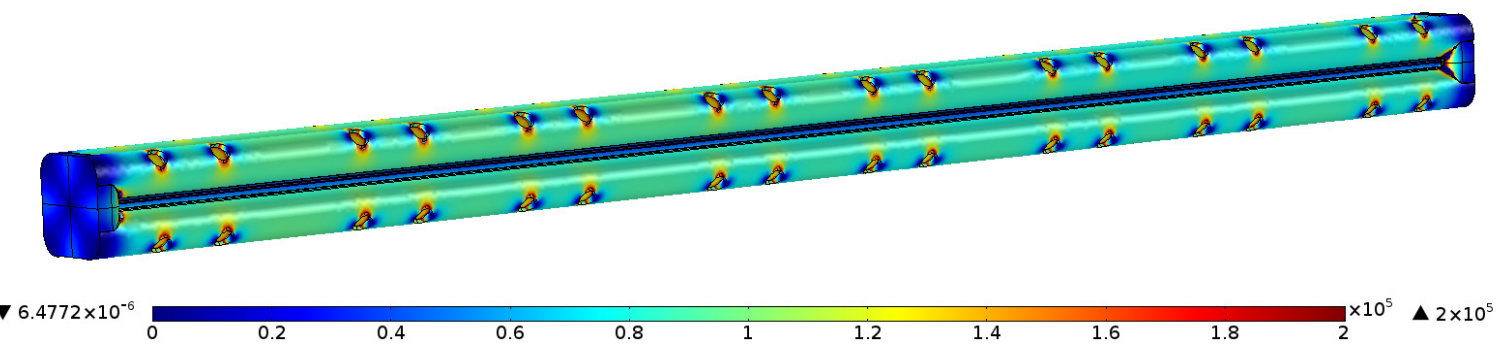

Figure 13. Peak power losses $\left(\mathrm{W} / \mathrm{m}^{2}\right)$ in the surface of the RFQ cavity, with all the tuners in uniform penetration. The model shown is a simplified model with no pumping pick-up ports included, but with modulation in full detail.

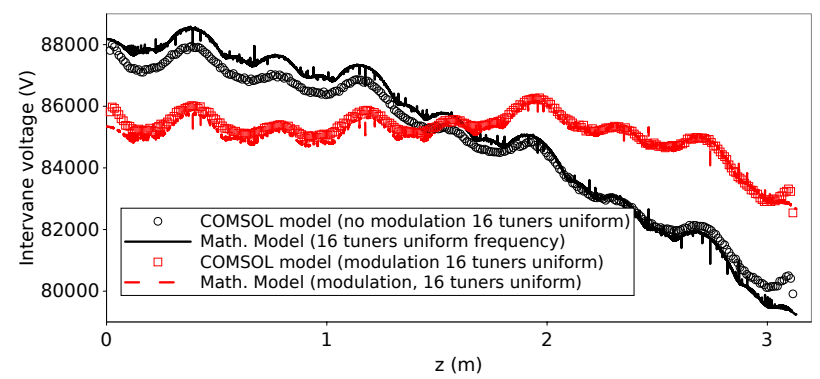

Figure 14. Comparison of the innervate voltage computed using the transmission-line model (full lines) and the voltages extracted from the FEM model of the whole length of the RFQ (open markers). In one situation the vane modulation is not considered and in the other the full details are included. The overlapping of the FEM and the mathematical model curves is very good.

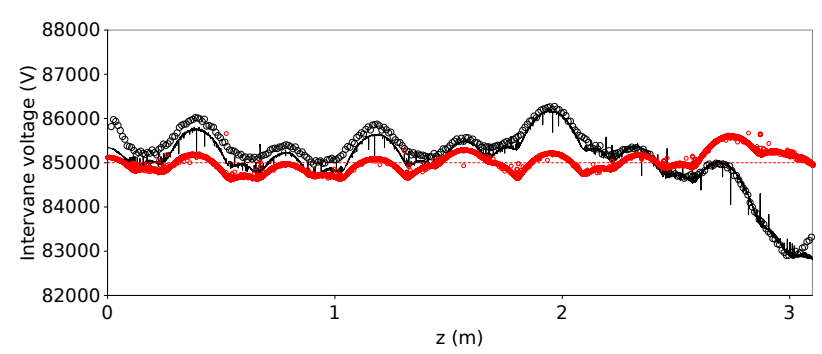

Figure 15. An optimized voltage profile (in red) as compared to the original one without optimization (black).

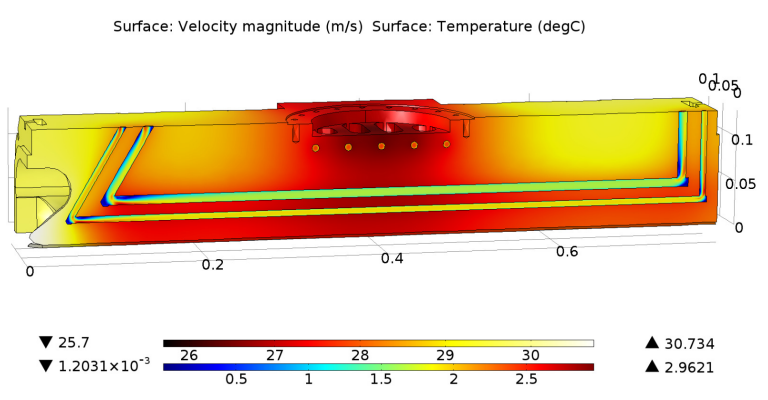

Figure 16. Temperature distribution in the solid and water velocity in the cooling channels, obtained from the thermo-mechanical model of the first segment. Input power corresponds to a duty cycle of $5 \%$. Water enters all the channels at $25^{\circ} \mathrm{C}$.

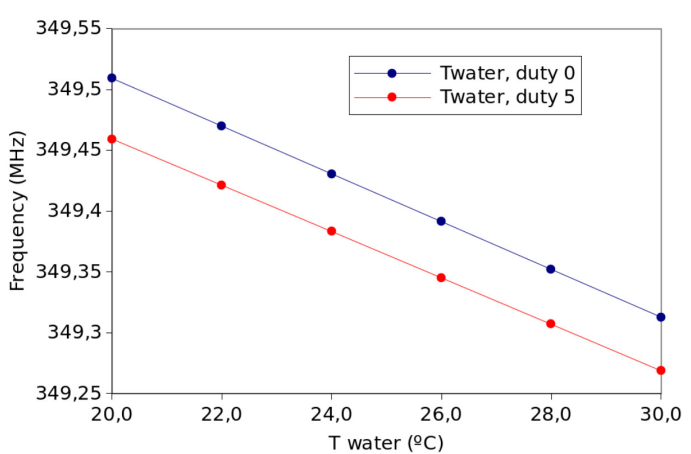

Figure 17. Change in cavity frequency with cooling water temperature, for two different RF power conditions. The results come from steady-state simulations.

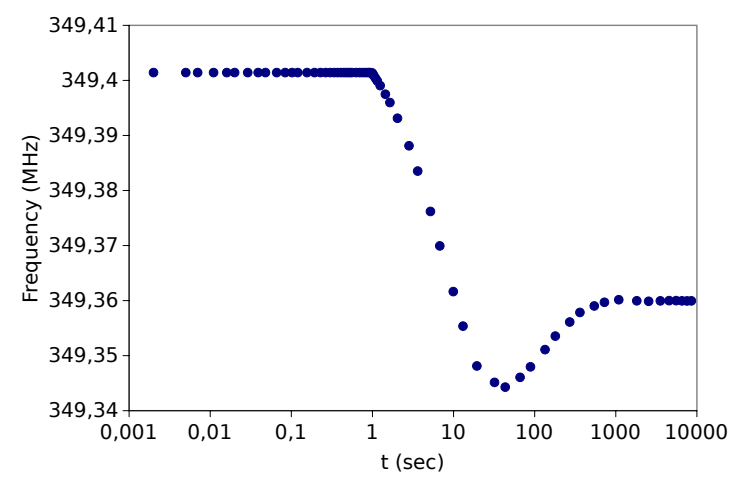

Figure 18. Frequency of the RFQ cavity (first segment) during a transient steps, from zero RF power to operation power at duty cycle $5 \%$ (power up). Cavity frequency is computed at all times of the simulation.

available for fabrication of the vanes. Each segments is an assembly of four elements, 2 major and 2 minor vanes, assembled together by using polymeric vacuum gaskets instead of brazing or other welding system. Material is copper OHFC (Cu 10100) quality.

\subsection{Mechanical model}

Starting from the electromagnetic design of the RFQ cavity a CAD model of the structure was built. The ports for tuners, vacuum grid and all other mechanical features were 


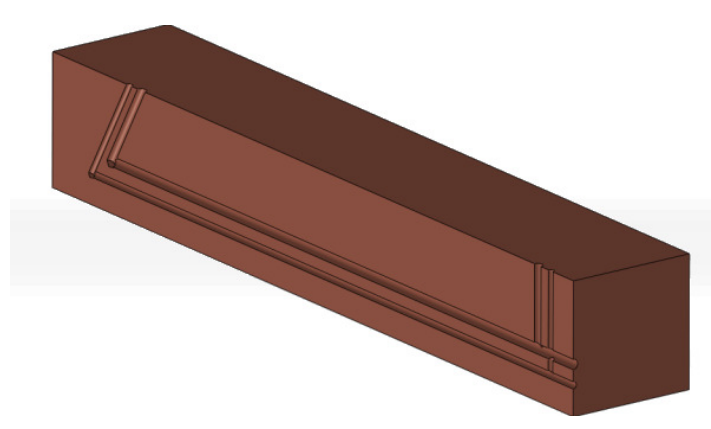

Figure 19. Model showing the deep drilling of the longitudinal cooling channels.

implemented in the model. RF and thermo-mechanical simulations were then ran again to validate the design. As described in a previous section, the vane modulations solids were built up using a home made CAD tool to avoid certain issues with the interpolation of the modulation curve[22].

\subsection{Fabrication steps}

\subsubsection{Raw material}

The material for the fabrication of the RFQ was supplied in blocks of two different sizes: 270x140 mm for major vanes and $115 \times 140 \mathrm{~mm}$ for the minor ones. Length of all blocks is $830 \mathrm{~mm}$. The copper grade selected is $\mathrm{Cu}$ OFE C10100.

\subsubsection{Squaring and deep drilling}

The first step in the fabrication process is to evenly square the copper blocks, to assure that each face is parallel to the opposing one and perpendicular to the others. Marks to fix the position of the cooling channels are machined in the corresponding faces.

A first rough machining is done, leaving an excess of about $2 \mathrm{~mm}$, followed by a stress-relieve annealing. The deep drilling of the longitudinal cooling channels is then done. This is made during the first steps of the fabrication process, and channels will then serve as reference for the rest of the geometry. In this way, the effects of a possible deformation due to the machining is minimized as major machining is done afterwards. A model showing the channels is shown in Fig. 19. Fig. 20 shows a detail of the first fabricated vane after this stage.

At this stage, the two channels are connected and open in the back side (higher energy) of the RFQ segment. They will be sealed in a later step.

\subsubsection{EBW of the cooling channels plugs}

Cooling channels are then sealed by inserting copper plugs and welding them. This is carried out by our staff at ESSBilbao's Advanced Welding Facility by means of electron beam welding (EBW). Three plugs are inserted and welded, one to separate the two channels and the other

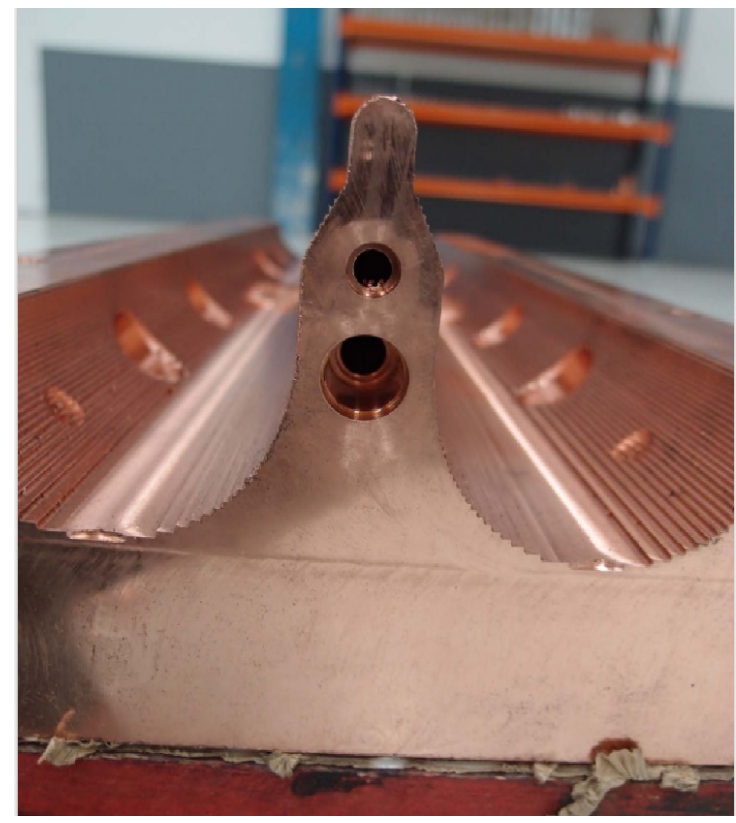

Figure 20. A major vane after rough machining. The holes of the cooling channels can be seen in the vane.

two to seal them from the external face (Fig. 21). In the final steps of the process this face will be machined and no external evidence of the welding of the plugs will be visible.

\subsubsection{Fine machining}

The last fabrication step for a vane is the fine machining, where all the final details are included. Particularly, the vane modulation needs a careful process in a temperature controlled machine to avoid over-heating that could give rise to deformations. Fig. 22 shows a major vane in the milling machine.

The milling of the modulation is made using a CAM controlled, 3-axes, HERMLE C800V machining center. Many displacements of the tool on the copper surface are used, removing a very thin layer each step. This increases milling time but also provide excellent surface quality (roughness $0.8 \mathrm{Ra}$ and mechanical tolerances around 0.005 $\mathrm{mm}$.

\subsubsection{Metrology}

The final step is the metrology of the fabricated piece (Fig. 23). Special care is taken in the measurement of the vane modulation profile. After control of the first major vane, a deviation of the modulation measured with respect to the design one was detected, despite the attention paid to the machining process. To correct this, a second machining of the modulation profile was performed, lowering the height from the bottom face by $100 \mathrm{um}$. The contact faces between major vanes and the two minor vanes were also machined removing the same height of material, so the four vane tips have the correct distribution after assembly.

Apart from this issue, the metrology results of the first vane of the first segment is very satisfactory (Fig. 24). 


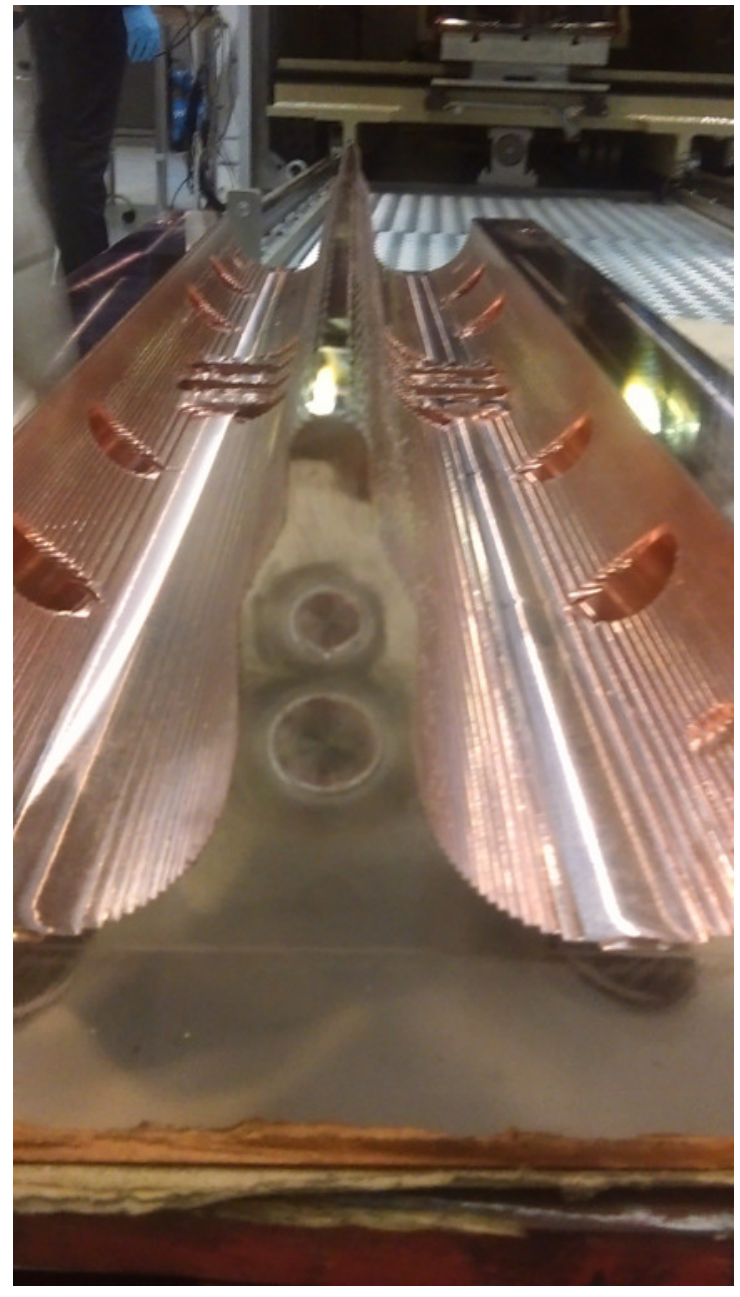

Figure 21. A major vane after cooling channels plugs have been inserted and welded by EBW.

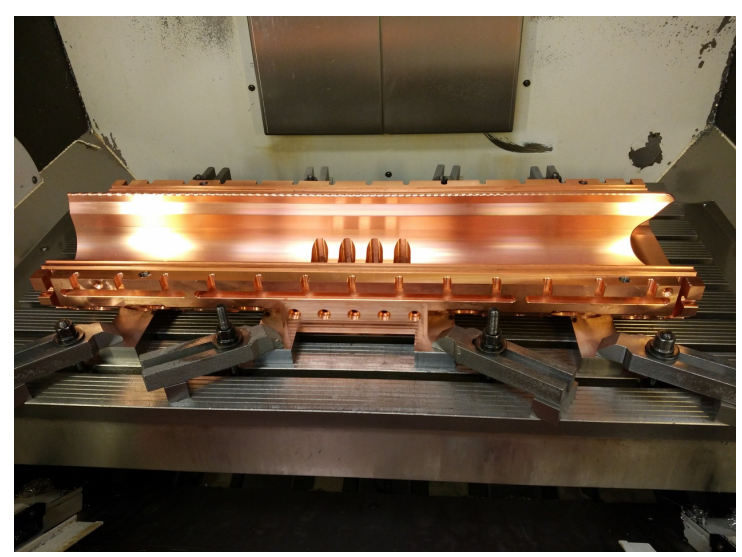

Figure 22. A major vane of the RFQ after fine machining in the milling machine.

\subsection{Assembly and vacuum strategy}

The vacuum strategy for the cavity is based on the use of polymeric vacuum gaskets at the unions between majorminor vanes, and also on the contact faces between the assembly of four vanes and the cover or inter-segment ring. RF contact seals (so-called finger strips) are considered for all contacts between surfaces. Once the four vanes are assembled (as in Fig. 1) and the alignment verified, the groove for the O-ring in the front side will be machined. This approach will allow assembling or disassembling the RFQ in case of misalignment or other problems. This strategy will be thoroughly tested with the first segment, in terms of vacuum levels and other issues. If results are satisfactory, the same procedure will be used for the rest of the segments. Otherwise, the strategy will be revisited and brazing of vanes will be considered. The results of these tests (expected for the last quarter of 2019) will be presented in future UCANS meetings.

\section{CONCLUSIONS}

ESS-Bilbao RFQ design has been summarized, including details on the beam dynamics, cavity electromagnetic design, cooling and thermo-mechanical studies. Fabrication of the first segment of the RFQ is ongoing. The whole segment will be received, and tests performed on it, before the end of 2019.

\section{References}

[1] ESS-Bilbao, ESS-Bilbao (2017), http://www . essbilbao.org

[2] ESS, European Spallation Source ERIC (2017), https://europeanspallationsource.se/

[3] Z. Izaola, I. Bustinduy, J. Corres, D. de Cos, C. de la Cruz, G. Harper, R. Miracoli, J. Muñoz, I. Rueda, A. Vizcaino et al., Advances in the Development of the ESS-Bilbao Proton Injector, in Proceedings of HB2016 (Malmo, Sweden, 2016), TUPMLY01, http://accelconf.web.cern.ch/ AccelConf/hb2016/papers/tupm1y01.pdf

[4] A. Letchford et al., Status of the RAL front end test stand, in Proceedings of IPAC 2015 (Richmond, VA, USA, 2015), THPF105, http: //accelconf.web.cern.ch/AccelConf/ IPAC2015/papers/thpf105.pdf

[5] C. Rossi et al., The Radiofrequency Quadrupole Accelerator for the LINAC4, in Proceedings of LINAC'08 (Victoria, BC, Canada, 2008), MOP040, http: //accelconf.web.cern.ch/accelconf/ linac08/papers/mop040.pdf

[6] N. Misiara et al., Recent $R F$ and mechanical developments for the ESS RFQ, in Proceedings of the LINAC2016 Conference, THPLR054 (2016), https://accelconf.web.cern.ch/accelconf/ IPAC2016/papers/thplr054.pdf 


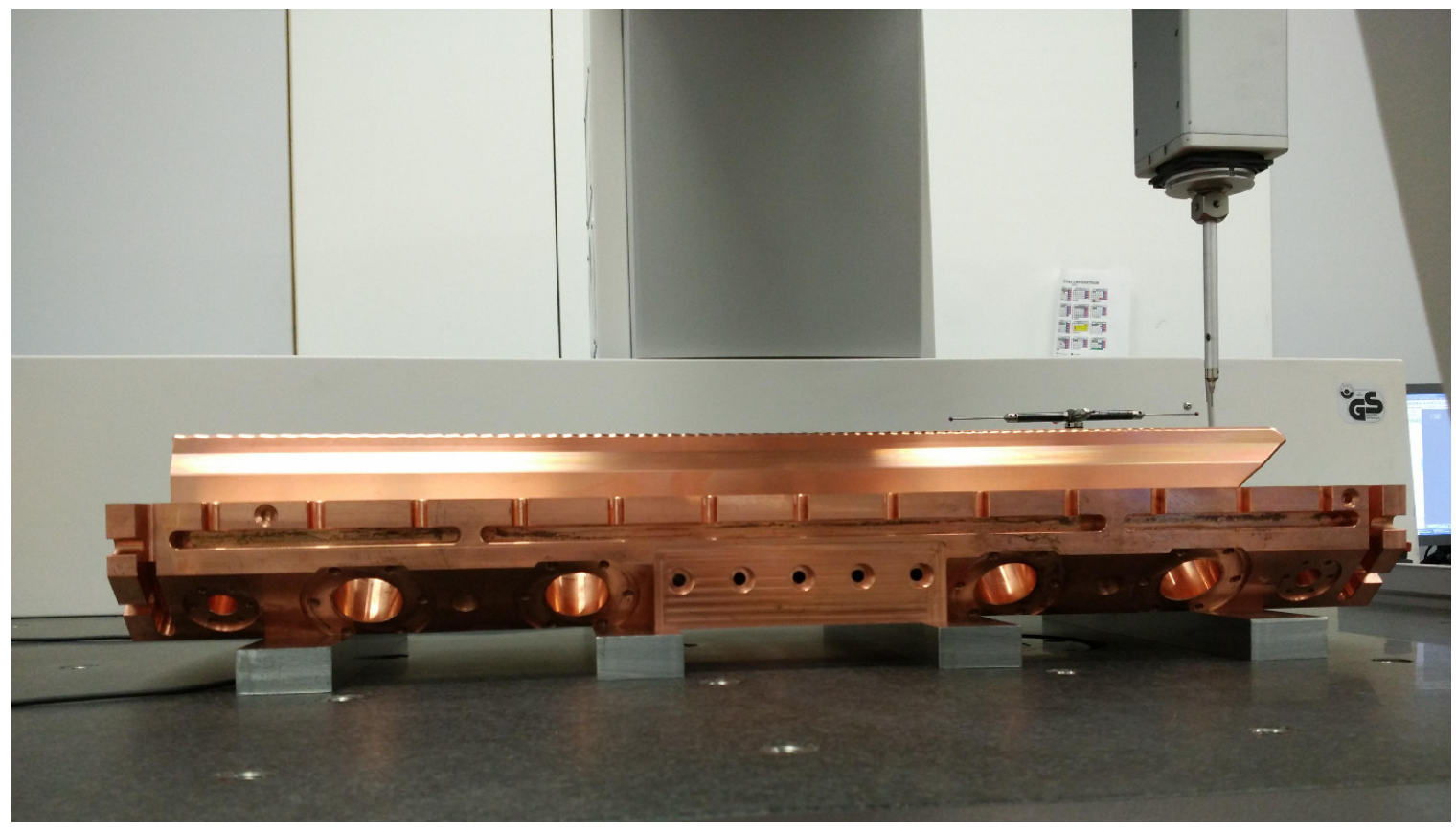

Figure 23. Major vane in the metrology station.

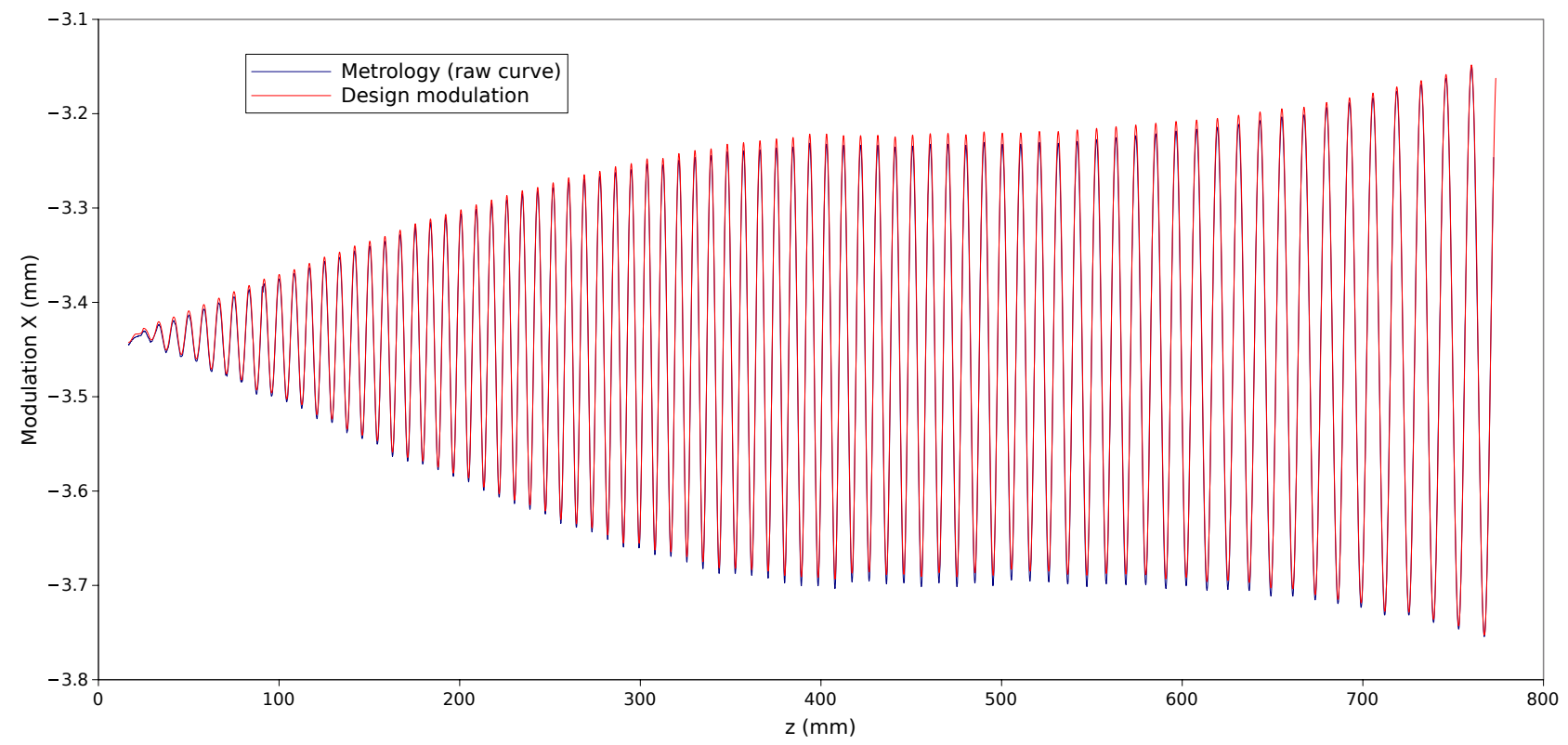

Figure 24. Comparison of measured and designed modulations.

[7] R. Ferdinand et al., Status Report on the 5 Mev Iphi RFQ, in Proceedings of the XX International Linac Conference (Monterey, California, 2000), http://accelconf.web.cern.ch/ AccelConf/100/papers/TUDQ4.pdf

[8] I. Bustinduy, J.L. Muñoz, eds., Technical Design Report: ESS-Bilbao RFQ (2015), ISBN 978-84-616-5445-1, https://www.essbilbao.org/st_docs/ technical-design-report-ess-bilbao-rfq
[9] J. Staples, S. Abbott, R. Caylor, R. Gough, D. Howard, R. MacGill, The Radiofrequency Quadrupole Accelerator for the LINAC4, in Proceedings of 1988 Linear Accelerator Conference (Williamsburg, Virginia, USA, 1988), http: //accelconf. web.cern. ch/accelconf/ linac08/papers/mop040.pdf

[10] O. Delfierrea, M. Desmons, A. France, R. Ferdinand, A new RF tuning method for the end regions of the IPHI 4-vane RFQ, in Proceedings of the EPAC 2006, MOPCH105 (Edinburgh, Scotland, 2006), https: //accelconf.web.cern.ch/ 
accelconf/EPAC2006/papers/mopch105.pdf

[11] L. Young, L. Rybarcyk, Tuning the LEDA RFQ 6.7 MeV accelerator, in Proceedings of the International LINAC Conference, 1998, MO4088 (Chicago, Illinois, 1998), accelconf.web.cern. ch/AccelConf/198/PAPERS/M04088.PDF

[12] I.M. Kapchinsky, V.A. Tepliakov, Tech. rep., Stanford Linear Accelerator Center (1969), sLACTRANS-0099

[13] A. Letchford, A. Schempp, A comparison of 4-rod and 4-vane RFQ fields, in Proceedings of EPAC 98 Conference (Stockholm, Sweden, 1998)

[14] T. Wangler, RF Linear Accelerators, Physics textbook (Wiley, 2008), ISBN 9783527406807, http: //books.google.es/books?id=5E04\_nyndUMC

[15] M.J. Browman, L. Young, Coupled RadioFrequency Quadrupoles as Compensated Structures, in Proceedings of LINAC1990 (Albuquerque, USA, 1990), p. $70, \mathrm{a}-12004-\mathrm{C}$

[16] General Particle Tracer, Pulsar Physics (2011), www. pulsar.nl/gpt

[17] R. Duperrier, Ph.D. thesis, University of Orsay, Orsay (2000)
[18] R. Ferdinand, R. Duperrier, J.M. Lagniel, P. Mattei, S. Nath, Tuning the LEDA RFQ $6.7 \mathrm{MeV}$ accelerator, in Proceedings of the International LINAC Conference, 1998, MO4029 (Chicago, Illinois, 1998), accelconf.web.cern.ch/AccelConf/ 198/PAPERS/M04029.PDF

[19] I. Bustinduy, J.M. et al., Design and Fabrication of the ESS-Bilbao RFQ Prototype Models, in Proceedings of IPAC 2012, THEPPB001 (New Orleans, Luisiana, USA, 2012), https: //accelconf. web.cern.ch/accelconf/ IPAC2012/papers/theppb001.pdf

[20] J. Muñoz, D. de Cos, I. Madariaga, I. Bustinduy, Design of ESS-Bilbao RFQ Linear Accelerator, in Proceedings of COMSOL Conference (2015)

[21] Open Cascade, Open Cascade Technology (2017), https://wwW . opencascade.com/

[22] J. Muñoz, I. Bustinduy, J. Martin, A. Ortega, I. Rueda, A. Zugazaga, M. Carrera, A. Garbayo, Mechanical fabrication of ESSBilbao RFQ, in Proceedings of IPAC 2017, THPIK083 (Copenhagen, Denmark, 2017), https://accelconf.web.cern.ch/accelconf/ IPAC2017/papers/thpik083.pdf 